Trauma Berufskrankh 2012 - 14[Suppl 2]:222-223 DOI 10.1007/s10039-011-1766-4

Online publiziert: 5. August 2011

(c) Springer-Verlag 2011

I. Pericie

Unfallkrankenhaus Berlin (ukb), Berlin

\title{
Sturzprävention im Krankenhaus und Pflegeheim
}

Psychische Folgen. Ein zentrales Thema hierbei stellt die Angst dar. Zum einem ist die Angst vor dem Stürzen eine wesentliche Sturzursache und zum anderen begünstigt sie den Verlust des Vertrauens in die eigene Mobilität, was zu einer massiven Einschränkung der Lebensqualität des Betroffenen führen kann $[1,4]$.

Ökonomische Folgen. Die ökonomischen Folgen für die Krankenhäuser und Pflegeheime spiegeln sich in resultierenden Imageschäden, haftungsrechtlichen Konsequenzen und erhöhten Kosten wider.

\section{Sturzprävention}

Stürze sind keine unvermeidbaren Schicksalsschläge. Mit einer konsequenten Sturzprävention können sie nachhaltig vermieden werden. Hierbei ist von einer umfassenden, individuellen Sturzprävention auszugehen, welche viel mehr beinhaltet, als freiheitsbeschränkte Maßnahmen zu verordnen. Sie ist gekennzeichnet durch [4]:

- das Erfassen von individuellen Sturzund Sturzfolgerisiken,

- das Analysieren der jeweiligen Ressourcen und

- dem Ableiten von multimodalen Maßnahmen.

Dabei ist die Individualität jedes Einzelnen unbedingt zu berücksichtigen.

\section{Expertenstandard}

Physische Folgen. Hierzu zählen beispielsweise Mortalität, hüftnahe Frakturen und Immobilität.
Den 3 oben genannten Ebenen der Sturzprävention wird der Nationale Experten- standard Sturzprophylaxe in der Pflege vom Deutschen Netzwerk für Qualitätsentwicklung in der Pflege (DNQP) [1] gerecht. Aus diesem Grund wird der Aufbau nationaler Expertenstandards grob vorgestellt.

Expertenstandards verstehen sich als ein evidenzbasiertes Instrument, mit dessen Hilfe Qualität von Leistungen definiert, eingeführt und bewertet werden kann. Sie unterstützen die Pflegenden, ihre hohe Verantwortung gegenüber den Pflegebedürftigen gerecht zu werden. Sie spiegeln den aktuellen Stand der Pflegeforschung und -wissenschaft wider.

Expertenstandards sind nach Donabedian [2] in Struktur-, Prozess- und Ergebnisqualität unterteilt und beinhalten verschiedene Ebenen, welche die jeweiligen Einrichtungen erfüllen sollen.

Jeder Expertenstandard verfügt über eine Standardaussage, die Standardaussage des Expertenstandard Sturzprophylaxe in der Pflege lautet:

„Jeder Patient/Bewohner mit einem erhöhten Sturzrisiko erhält eine Sturzprophylaxe, die Stürze verhindert oder Sturzfolgen minimiert."

Um dieser großen Anforderung gerecht $\mathrm{zu}$ werden, wurden die Ebenen mit folgenden Inhalten gefüllt:

- Die erste Ebene stellt die Identifikation des Sturzrisikos dar.

- Die zweite Ebene fordert die Information und Beratung des Patienten bzw. Bewohners bezüglich der individuellen Sturzrisikofaktoren und der geeigneten Interventionen.

- In der dritten Ebene wird die Erstellung des Maßnahmenplans festgehal- 
ten [1]. Beispielhaft werden anschließend besondere Maßnahmen genannt:

- Balance- und Kräftetraining,

- angemessene, gut funktionierende Hilfsmittel,

- Umfeldgestaltung und

- Transfertechniken.

- Die Gewährleistung der Maßnahmen für die Sturzprävention wird mit der vierten Ebene eingefordert.

- Die Informationsweitergabe über das Sturzrisiko an alle an der Versorgung Beteiligten ist in der fünften Ebene festgehalten.

- Die sechste und letzte Ebene des Expertenstandards widmet sich der systematischen Sturzerfassung und -analyse.

Zusammengefasst versteht sich der Expertenstandard Sturzprophylaxe in der Pflege als eine Sammlung von Zielaussagen, welche in Struktur-, Prozess- und Ergebnissaussagen unterteilt sind und sich auf die Pflegefachkraft, die Einrichtung und den Patienten bzw. Bewohner beziehen. Zudem wird eine Reihe von Maßnahmen zur Sturzprophylaxe angeboten.

\section{Fazit für die Praxis}

Der nationale Expertenstandard Sturzprophylaxe in der Pflege bietet ein umfassendes Instrument zur Vermeidung von Stürzen im Krankenhaus und Pflegeheim.

Insbesondere die multifaktoriell angelegte Sturzprävention findet hier Berücksichtigung und unterstützt die Pflegenden adäquat, eine individuelle Risikoanalyse zu erfassen, Ressourcen zu analysieren, daraus multimodale Maßnahmen abzuleiten und somit Stürze im Krankenhaus und Pflegeheim zu vermeiden.

\section{Korrespondenzadresse}

\section{Pericie}

Unfallkrankenhaus Berlin (ukb), Warener Straße 7, 12683 Berlin ines.pericie@ukb.de

Interessenkonflikt. Der korrespondierende Autor gibt an, dass kein Interessenkonflikt besteht.

\section{Literatur}

1. Deutsches Netzwerk für Qualitätsentwicklung in der Pflege (2006) Expertenstandard Sturzprophylaxe in der Pflege. Deutsches Netzwerk für Qualitätsentwicklung in der Pflege, Osnabrück

2. Donabedian A (1966) Evaluating the quality of medical care. Milbank Memorial Fund Quarterly XLIV(3),2

3. Funk M, Pierobon A (2007) Sturzprävention bei älteren Menschen: Risiken - Folgen - Maßnahmen. Thieme, Stuttgart New York

4. Tideiksaar R (2000) Stürze und Sturzprävention: Assessment - Prävention - Management. Huber, Bern Stuttgart Toronto
Trauma Berufskrankh 2012 - 14[Suppl 2]:222-223

DOI 10.1007/s10039-011-1766-4

(c) Springer-Verlag 2011

\section{Pericie}

\section{Sturzprävention im Krankenhaus und Pflegeheim}

\section{Zusammenfassung}

Stürze sind vermeidbar. Mit einer gut gestalteten Sturzprävention im Krankenhaus und Pflegeheim lassen sie sich nachweislich verringern. Dabei werden die Maßnahmen so konzipiert, dass die Faktoren für ein Sturzrisiko minimiert werden können. Ein wesentliches Instrument auf der Grundlage der aktuellen Pflegeforschung ist der nationale Expertenstandard Sturzprophylaxe in der Pflege. Mit seiner Hilfe ist eine adäquate, multidimensionale und systematische Sturzprophylaxe möglich. Er ist für die Versorgung sowohl im Krankenhaus als auch im Pflegeheim gültig und anwendbar.

\section{Schlüsselwörter}

Stürze · Prävention · Krankenhaus .

Pflegeheime $\cdot$ Expertenstandard

\section{Hospital and nursing home fall prevention}

\section{Abstract}

Falls can be prevented. Evidence indicates that they can be reduced by a well-conceived fall prevention program in hospitals and nursing homes. The measures are designed in such a fashion that risk factors for falls can be minimized. An important instrument based on current healthcare research is the national expert consensus guideline on fall prevention in nursing care which helps to ensure that adequate, multidimensional, and systematic fall prevention is possible. It applies to both hospital and nursing home care.

\section{Keywords}

Falls, accidental · Prevention · Hospital ·

Nursing homes - Expert consensus guidelines 